\title{
Occult HIV infection in a large sample of health-care users in Lombardy, Italy in 2014-2015: implications for control strategies
}

\author{
L. SCUDELLER ${ }^{1} *$ F. GENCO ${ }^{2}$, F. BALDANTI ${ }^{2,3}$, G. COMOLLI ${ }^{2,4}$, \\ G. ALBONICO ${ }^{5}$, M. PRESTIA ${ }^{2}$ AND V. MERONI ${ }^{2,6}$ \\ ${ }^{1}$ Clinical Epidemiology Unit - Scientific Direction, IRCCS Policlinic San Matteo Foundation, Viale Golgi 19, \\ 27100 Pavia, Italy \\ ${ }_{3}^{2}$ Microbiology and Virology Unit, IRCCS Policlinic San Matteo Foundation, Pavia, Italy \\ ${ }^{3}$ Section of Microbiology, Department of Clinical, Surgical, Diagnostic and Paediatric Sciences, University of \\ Pavia, Pavia, Italy \\ ${ }^{4}$ Experimental Research Laboratories, Biotechnology Area, IRCCS Policlinic San Matteo Foundation, Pavia, Italy \\ ${ }^{5}$ Department of Clinical Chemistry, IRCCS Policlinic San Matteo Foundation, Pavia, Italy \\ ${ }^{6}$ Department of Internal Medicine and Clinical Therapy, University of Pavia, Pavia, Italy
}

Received 8 November 2016; Final revision 13 April 2017; Accepted 16 May 2017;

first published online 22 June 2017

\section{SUMMARY}

We estimated the number of people unaware of their human immunodeficiency virus (HIV) infection in our province, Pavia (population 540000 ) in Lombardy, Italy, by means of anonymous unlinked testing of 10044 serum/plasma samples residual from clinical analyses at the outpatient clinic of Policlinico San Matteo in 2014 and 2015. Ethical and legal approval was obtained prior to study start. Samples were irreversibly anonymised, only retaining gender and 5-year age class. Five sample pools were tested for HIV using LIAISON ${ }^{\circledR}$ XL MUREX HIV $\mathrm{Ab} / \mathrm{Ag}$ (DiaSorin, Saluggia, Italy). If the pool tested positive, individual samples underwent confirmatory tests, Innotest HIV Antigen mAb (Fujirebio Europe, Gent, Belgium) and HIV BLOT $2 \cdot 2$ (MP Diagnostics, Singapore). Among the 10044 samples processed, eight were confirmed positive $(0 \cdot 08 \%, 95 \%$ confidence interval $0 \cdot 03-0 \cdot 16 \%)$, all were males and age was $>50$ in $3(37 \cdot 5 \%)$. If projected to the entire population of the Pavia province, this would result in approximately 1000 people unaware of their HIV infection, with age older than expected. In Italy, HIV testing is voluntary, universally free-of-charge and (upon request) anonymous. Nevertheless, this study demonstrates that it is suboptimally employed, and that new strategies and population-level actions will be needed to achieve better implementation of HIV testing and HIV control in our province.

Key words: Anonymous unlinked HIV testing, ethical issues, human immunodeficiency virus - HIV, infection control, occult HIV infection, public health policy, seroprevalence, surveillance.

\section{INTRODUCTION}

Estimates of number and proportion of people with undiagnosed human immunodeficiency virus (HIV)

\footnotetext{
* Author for correspondence: L. Scudeller, Clinical Epidemiology Unit - Scientific Direction, IRCCS Policlinic San Matteo Foundation, Viale Golgi 19, 27100 Pavia, Italy.

(Email: 1.scudeller@smatteo.pv.it)
}

are important for implementing public health strategies. In particular, persons unaware of their HIV infection do not benefit from early treatment and can contribute up to $50-90 \%$ of new HIV infections, mainly (but not only) in the acute phase [1-3].

In 2008, the Italian Ministry of Health instituted a surveillance system, adding HIV infection (acquired immunodeficiency syndrome (AIDS) surveillance started 
on 1987) to the list of Class III infections to be notified [4]. In 2007, incidence of new HIV type 1 (HIV-1) diagnoses in Italy was estimated 7.1 per 100000 population, and remained stable thereafter, and in 2013, it was 6.0 per 100000 [5].

According to the UNAIDS guidelines, notification of new HIV cases should be paralleled by unlinked HIV prevalence monitoring through HIV testing of serum from blood drawn for reasons other than HIV testing [6]. In 2006, Centers for Disease Control and Prevention (CDC) recommended screening patients aged 13-64 years for HIV infection in health-care settings that have a prevalence of undiagnosed HIV infection of $>0 \cdot 1 \%$ [7].

The overall aim of the study was to estimate the (unknown) HIV seroprevalence in the general population of Pavia province of Italy. The primary outcome was the estimate of occult nosocomial seroprevalence (ONS), using anonymous and unlinked blood samples among residents accessing health services.

\section{METHODS}

A survey, by means of anonymous and unlinked HIV testing of serum samples drawn for reasons other than HIV testing, was designed and performed from January 2014 to December 2015. Approval of the Institutional Review of Istituto di Ricovero e Cura a Carattere Scientifico (IRCCS) Policlinic San Matteo Foundation Board was obtained prior to study start. The detailed study protocol is available upon request.

\section{Setting}

Pavia is a small (population ca. 70000 ) university town, $30 \mathrm{~km}$ south of Milan, with a large teaching and research hospital and a very ancient university with medical school. The province of Pavia has a population of ca. 550000 inhabitants and an area of $2968.64 \mathrm{~km}^{2}$. It is located in the Lombardy region, the most densely populated region in Italy, with 10 million inhabitants [8], accounting for $17 \%$ of the total Italian population.

As of 31/12/2012, there were 29508 people living with HIV/AIDS (PLWH) in the Lombardy region (prevalence 328/100 000); almost $45 \%$ are females [9] (Table 1). In 2013, new HIV diagnoses in Lombardy have been 931 (incidence 10.5/100000), of which $84 \%$ were males, $18 \%$ of foreign origin and only $5 \%$ were intravenous drug users (IVDUs); $55 \%$ were late presenters (CD4+ cell count $<350 / \mathrm{mmc}$ ); and $2 / 3$ of
Table 1. Selected epidemiological characteristics of HIV infection and AIDS in Lombardy region and Pavia province, Italy

\begin{tabular}{lll}
\hline \hline & Lombardy (2013) & Pavia (2015) \\
\hline Population & 10 millions & 550000 \\
PLWH & 29508 & 1276 \\
$\quad$ Prevalence (per 100 000) & 328 & 215 \\
$\quad$ Males & $67 \%$ & $70 \%$ \\
New HIV diagnoses & 931 & 26 \\
$\quad$ Incidence (per 100 000) & $10 \cdot 5$ & $9 \cdot 4$ \\
$\quad$ Males & $84 \%$ & $81 \%$ \\
Median age (males) & 38 years & 50 years \\
Median age (females) & 35 years & 47 years \\
\hline \hline
\end{tabular}

HIV, human immunodeficiency virus; AIDS, acquired immunodeficiency syndrome; PLWH, people living with HIV/AIDS.

these were in advanced stage of HIV infection (CD4+ <200/mmc). New AIDS cases were 284 (with a downward trend parallel to the national incidence) [5, 10, 11]. In 2015, new HIV diagnoses in Lombardy were $815(8 \cdot 1$ per 100000$)$ [10].

In Pavia province, as of $31 / 12 / 2015$, there were 1276 PLWH (prevalence 215/100 000); males are 70.4\%. New diagnoses of HIV infection in 2008 have been $116(21 \cdot 5 / 100000$, around three times higher than the national average); in 2013, 51 (40 males and 11 females); in 2014, 47 (35 males and 12 females); and in 2015, 26 (21 males and 5 females) (data from the Pavia Local Health Authority).

The surveillance system of blood donors (a population at very low risk of HIV) in 2008 detected, among the 21777 donors in Lombardy, three HIV-infected subjects $(1 \cdot 4 / 10000)$. One of these was resident in Pavia (1/8575 Pavia donors, 1·2/10 000).

\section{Eligibility criteria}

All residual serum or plasma samples following processing for diagnostic purposes were collected at the Laboratorio Analisi Chimico-Cliniche of the IRCCS Policlinic San Matteo Foundation during the study period and included in the study. Duplicate samples from patients accessing the hospital within 6 months were excluded from the study.

Only samples from patients residing in the city of Pavia or in Pavia province were considered in the study.

Of note, at Policlinico San Matteo, HIV patients perform routine blood tests at the outpatient clinic of the Infectious Diseases Institute or of the Division 
of Tropical Diseases during routine periodic workup. Therefore, no mix-up of samples from patients with known HIV infection with those from patients with potentially undiagnosed HIV infection could have occurred since they were collected at different outpatient clinics.

\section{Study procedures}

Samples were collected weekly at the Virology Unit after checking for duplicates within the previous 6 months and were irreversibly anonymised (the only information retained was gender, 5-year age group and calendar month of sampling). The residual plasma or serum was aliquoted into two to three vials, and stored at $+4{ }^{\circ} \mathrm{C}$. To reduce time and cost, samples were pooled in five-patient pools and tested together. In case of pool positivity at screening tests, the five individual samples were tested individually by confirmatory tests.

Pools were tested for HIV using LIAISON ${ }^{\circledR}$ XL MUREX HIV Ab/Ag (DiaSorin, Saluggia, Italy). The LIAISON $^{\circledR}$ XL MUREX HIV Ab/Ag assay uses chemiluminescence immunoassay technology for the combined qualitative determination of p24 antigen of HIV-1 and specific antibodies to both HIV-1 (groups $\mathrm{M}$ and $\mathrm{O}$ ) and/or HIV type 2 in human serum or plasma samples. Confirmatory tests were Innotest HIV Antigen mAb (Fujirebio Europe, Gent, Belgium) and HIV BLOT 2.2 (MP Diagnostics, Singapore).

All positive samples were also tested with real-time PCR assay to investigate the presence of RNA virus. The real-time reverse transcriptase test was performed adopting the protocol established by the Working Group for HIV quantification (AC11) of the French National Agency for AIDS Research (ANRS). The test was conducted with the Generic HIV charge viral kit (Biocentric, Bandol, France).

All the tests were performed according to manufacturers' instructions.

\section{Sample size}

The working hypothesis for sample size calculation was ONS 100/100 000; to estimate it with $95 \%$ confidence intervals (CIs) between 48 and 183/100 000, we planned to test at least 10000 samples.

\section{Ethical issues}

In Italy, HIV testing is voluntary, universally freeof-charge and, upon request, anonymous. An ethical issue would arise for HIV-positive samples to which no linking back to original individual can occur; therefore, all patients undergoing blood sampling at the Fondazione have been reminded that upon request, HIV testing can be performed, with pre- and postcounselling, any day of the week at the Clinic of Infectious Diseases of the Fondazione.

Anonymous unlinked HIV testing for surveillance purposes has been used from the beginning of the epidemic in several countries, and is still used [12-20]. The World Health Organisation (WHO) included it into its second-generation surveillance tools $[6,21]$. Ethical issues have been reviewed by the WHO [22, 23].

Briefly, the guidance provided by the WHO is that, in the case of blood drawn for other purposes, communities should be broadly notified that blood collected for one purpose may be anonymously tested for HIV. Fully informed consent is not required, but the individual who wishes to opt out of such surveillance should be respected where possible. If the numbers of individuals opting out threaten the validity of surveillance efforts, ethics review committees will have to determine whether the public health significance of the studies warrants over-ride the right to refuse to participate [22].

In Italy in 2009, the specific Authority 'Garante per la Protezione dei Dati Personali' issued a regulation, which waives the need for informed consent in two situations: (a) the person ignores his condition, for example, research in which the information note on the study would result in the disclosure of information concerning the conduct of the study, which could cause material damage or psychological problems to those concerned; (b) logistical or organisational issues, such that excluding those patients who were not contacted for consent would introduce a bias in the study results [24].

\section{Statistics}

Descriptive statistics were obtained for the study variables. ONS (sample prevalence) was defined as number of confirmed positive HIV/number of tests at the clinical biochemistry laboratory in the study period, overall and by age and gender, with $95 \%$ CIs.

Projected estimated prevalence was calculated, by age and gender, on the population of Pavia province. Population data at 1 January 2014 and 1 January 2015 were obtained from the Italian National Statistical Institute (ISTAT) [25]. Projections were made for the point estimate and the lower and upper $95 \%$ confidence limits (CI) of sample prevalence. 
Table 2. Description of samples, positive samples, Pavia population and estimates of HIV prevalence, by gender and age group

\begin{tabular}{|c|c|c|c|c|c|c|c|c|c|c|c|}
\hline Year & Gender & $\begin{array}{l}\text { Age } \\
\text { group } \\
\text { (years) }\end{array}$ & $\begin{array}{l}\text { No. of } \\
\text { HIV- } \\
\text { positive } \\
\text { samples }\end{array}$ & $\begin{array}{l}\text { No. of } \\
\text { samples }\end{array}$ & $\begin{array}{l}\text { Sample } \\
\text { rate (per } \\
100000)\end{array}$ & $\begin{array}{l}\text { Lower } \\
95 \% \mathrm{CI} \\
\text { (per } \\
100000 \text { ) }\end{array}$ & $\begin{array}{l}\text { Upper } \\
95 \% \text { CI } \\
\text { (per } \\
100000 \text { ) }\end{array}$ & $\begin{array}{l}\text { Pavia } \\
\text { population }\end{array}$ & $\begin{array}{l}\text { Projected } \\
\text { HIV } \\
\text { cases on } \\
\text { population }\end{array}$ & $\begin{array}{l}\text { Lower } \\
95 \% \text { CI } \\
\text { projected } \\
\text { cases }\end{array}$ & $\begin{array}{l}\text { Upper } \\
95 \% \text { CI } \\
\text { projected } \\
\text { cases }\end{array}$ \\
\hline 2014 & Male & 0 & 0 & 48 & 0 & 0 & 7397 & 42627 & 0 & 0 & 3153 \\
\hline 2014 & Male & 18 & 0 & 58 & 0 & 0 & 6162 & 16852 & 0 & 0 & 1038 \\
\hline 2014 & Male & 25 & 1 & 197 & 508 & 13 & 2796 & 50772 & 258 & 7 & 1419 \\
\hline 2014 & Male & 40 & 3 & 434 & 691 & 143 & 2007 & 85985 & 594 & 123 & 1725 \\
\hline 2014 & Male & 60 & 1 & 524 & 191 & 5 & 1059 & 45459 & 87 & 2 & 481 \\
\hline 2014 & Male & 75 & 1 & 399 & 251 & 6 & 1388 & 24363 & 61 & 2 & 338 \\
\hline 2014 & Female & 0 & 0 & 64 & 0 & 0 & 5601 & 40235 & 0 & 0 & 2254 \\
\hline 2014 & Female & 18 & 0 & 95 & 0 & 0 & 3809 & 15912 & 0 & 0 & 606 \\
\hline 2014 & Female & 25 & 0 & 370 & 0 & 0 & 992 & 49804 & 0 & 0 & 494 \\
\hline 2014 & Female & 40 & 0 & 693 & 0 & 0 & 531 & 84385 & 0 & 0 & 448 \\
\hline 2014 & Female & 60 & 0 & 751 & 0 & 0 & 490 & 49355 & 0 & 0 & 242 \\
\hline 2014 & Female & 75 & 0 & 500 & 0 & 0 & 735 & 42577 & 0 & 0 & 313 \\
\hline 2015 & Male & 0 & 0 & 85 & 0 & 0 & 4247 & 42583 & 0 & 0 & 1809 \\
\hline 2015 & Male & 18 & 0 & 88 & 0 & 0 & 4105 & 16793 & 0 & 0 & 689 \\
\hline 2015 & Male & 25 & 2 & 343 & 583 & 71 & 2090 & 49238 & 287 & 35 & 1029 \\
\hline 2015 & Male & 40 & 0 & 666 & 0 & 0 & 552 & 87070 & 0 & 0 & 481 \\
\hline 2015 & Male & 60 & 0 & 636 & 0 & 0 & 578 & 45417 & 0 & 0 & 263 \\
\hline 2015 & Male & 75 & 0 & 498 & 0 & 0 & 738 & 25367 & 0 & 0 & 187 \\
\hline 2015 & Female & 0 & 0 & 120 & 0 & 0 & 3027 & 40179 & 0 & 0 & 1216 \\
\hline 2015 & Female & 18 & 0 & 154 & 0 & 0 & 2367 & 15823 & 0 & 0 & 375 \\
\hline 2015 & Female & 25 & 0 & 533 & 0 & 0 & 690 & 48185 & 0 & 0 & 332 \\
\hline 2015 & Female & 40 & 0 & 946 & 0 & 0 & 389 & 85406 & 0 & 0 & 332 \\
\hline 2015 & Female & 60 & 0 & 1030 & 0 & 0 & 358 & 49274 & 0 & 0 & 176 \\
\hline 2015 & Female & 75 & 0 & 803 & 0 & 0 & 458 & 43387 & 0 & 0 & 199 \\
\hline
\end{tabular}

HIV, human immunodeficiency virus; $95 \%$ CI, 95\% confidence interval.

Stata computer software version 14.0 (Stata Corporation, 4905 Lakeway Drive, College Station, Texas 77845, USA) was used for statistical analysis.

\section{RESULTS}

In the study period, 10044 samples were collected. The number of samples positive at screening test was 18 , and eight $(44 \%)$ were confirmed positive, for an overall ONS 79/100 000 (95\% CI 34-156). The positive samples all belonged to males, three in age group 30-35 years, one in age group 40-45, 45-50, $50-55,60-65,80-85$ years each; six were detected in 2014 and two in 2015.

Projected estimates on the entire Pavia population (Table 2) indicate that the potential number of people unaware of their HIV infection is 1000 (95\% CI 134-12 511 ) in 2014, and 287 (95\% CI 35-7088) in 2015 (Fig. 1).

The positive predictive value in this context was $44 \%$ (95\% CI 21-69), despite the high sensitivity and specificity of the test.
All eight positive samples at confirmatory test resulted negative at the PCR test.

\section{DISCUSSION}

In Italy, a total of 123000 individuals aged 15 or more (prevalence $0 \cdot 28 \%$ ) were estimated to be living with HIV/AIDS in Italy at the end of 2012, with males outnumbering females by almost 10 -fold [26]. These estimates were obtained with input from (among other sources) serosurveys among injecting drug users, men who have sex with men (MSM) and female sex workers [27], but none in a sample of the general population. Our study provides such an estimate.

\section{Main results}

Our project aimed at obtaining an estimate on HIV seroprevalence in the province of Pavia, based on persons accessing health-care services for reasons other than HIV, via anonymous and unlinked HIV testing. 


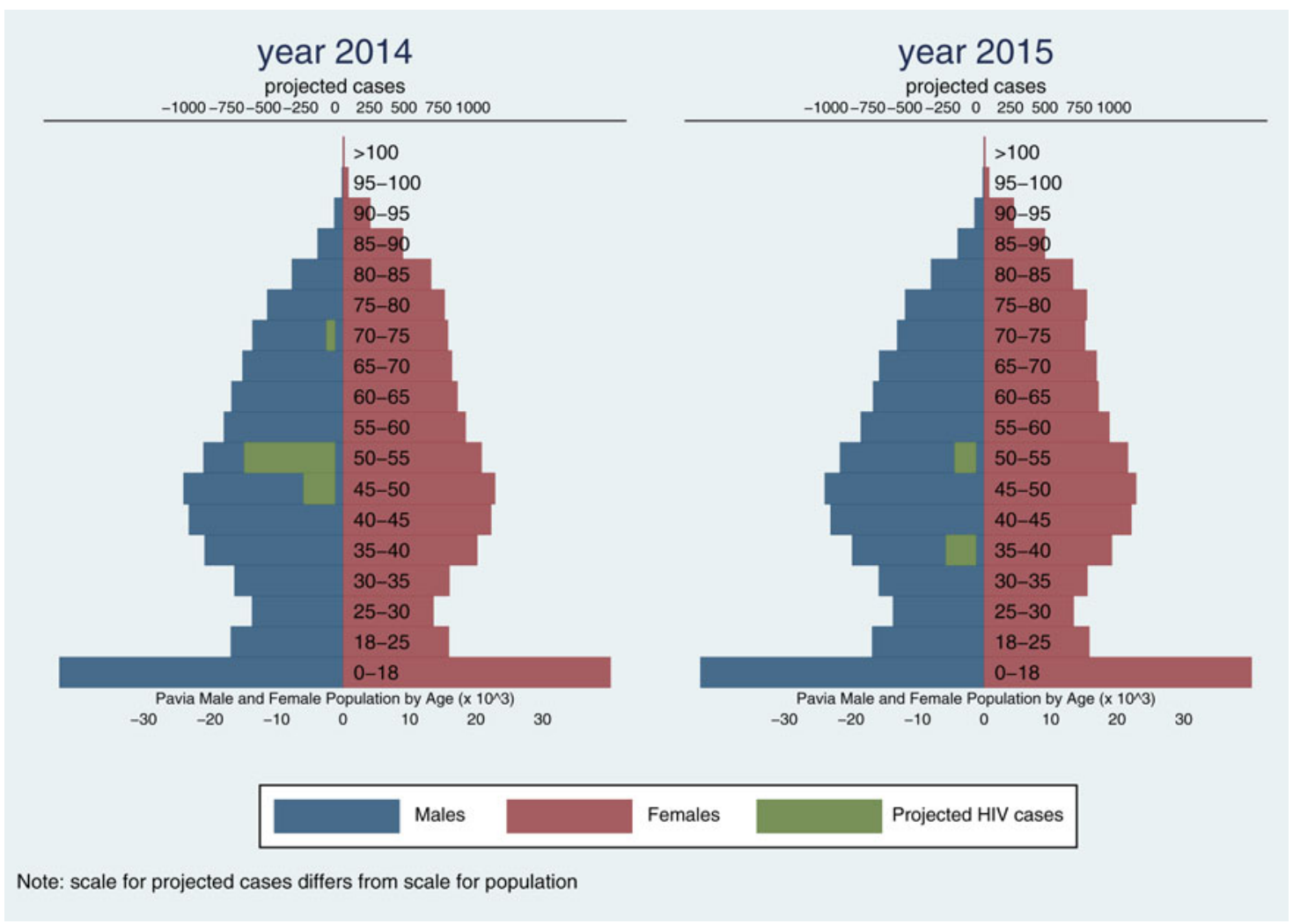

Fig. 1. Pavia population (by gender and age group) and projected estimates of people unaware of their HIV infections, in 2014 and 2015. HIV, human immunodeficiency virus.

We found a prevalence of HIV occult infection of approximately $0 \cdot 1 \%$; this direct measurement confirms estimates recently obtained by estimation methods in Italy [28]. We also estimate that the number of people unaware of their HIV infection is close to $50 \%$ of the known prevalence, and therefore that about one-third of PLWH in Pavia province is unaware of the infection. Considering the selection bias implicit in the anonymous unlinked testing of residual blood samples from a population accessing health care, this result agrees with the known new HIV diagnoses in Pavia province (51 in 2014 and 26 in 2015).

The socio-demographic picture of our province is similar to the whole of Lombardy [8], a region that contributes to the majority of HIV infection in Italy with a prevalence of diagnosed HIV cases of $0.3 \%$ [9]. Its proximity to Milan metropolitan area (population 3 millions) must also be taken into account in interpreting these findings.

\section{Research in context}

Almost 10 years ago, the estimated proportion of unknown HIV prevalence for Italy was 25\% [29], but our current results are in line with more recent estimates in other European contexts. In the Netherlands in 2012 , the estimate was $34 \cdot 2 \%$ (with $95 \%$ CI $21 \cdot 6$ 48.8) [30]. In Madrid, Spain, a serosurvey conducted in 2008-2009 found an unknown HIV prevalence of $0 \cdot 35 \%$ among 3695 subjects who agreed to participate. Of note, in that study, $31 \%$ of the subjects opted out of participation [31]. On the other hand, in the USA (a very different setting), recent estimated percentages of persons living with HIV who had received a diagnosis of HIV by the end of 2012 ranged from $77 \%$ in Louisiana to $\geqslant 90 \%$ in Colorado, Connecticut, Delaware, Hawaii and New York [32].

It is therefore evident that in our province and in the whole Lombardy, more efforts are needed to better implement a Test and Treat strategy, as supported by the WHO and UNAIDS several years ago [33].

Consequences of unawareness of HIV infection and late testing are well known: in 2013 in Italy, $68 \cdot 2 \%$ of AIDS diagnoses were made in patients never tested for HIV previously, the proportion of late presenters (CD4+ cell count $<350 / \mathrm{mmc}$ ) was $57 \cdot 6 \%$ and those in advanced stage of HIV infection (CD4+ $<200 / \mathrm{mmc}$ ) was $37 \cdot 6 \%[5]$. 
This implies that most patients with AIDS were not aware of their HIV status, were not offered highly active antiretroviral therapy (HAART) and could not be counselled to modify their risk behaviour. Another fraction of this population is composed of persons who, having completely neglected the issue, did not return for HIV testing results or did not accept the therapeutic consequences of a positive HIV test, for fear of paying a social cost or for lack of information of efficacy of HIV treatment.

Surveillance of new HIV diagnoses in Italy has been implemented since 2008, in addition to that for AIDS cases which started on 1987 [4]. In Italy, all health costs of HIV patients are entirely (even for conditions not linked to HIV) covered by the National Health System; $87.6 \%$ of the 94146 persons living with HIV-1 were on antiretroviral therapy in 2012 [9]. However, epidemiological data show that the HIV epidemic in Italy has not slowed down.

The successes of HAART therapy make both mandatory and urgent strategies of active HIV testing which, without forgetting the inalienable right of the individual to refuse diagnostic or therapeutic procedures, would allow to eradicate those situations where HAART is not offered because of unawareness or lack of adequate counselling. It is worth noting that the social stigma that the disease has always carried with itself still accompanies persons living with HIV/ AIDS and constitutes the main determinant of delay in diagnosing HIV infection. WHO guidelines on self-HIV testing are expected in 2016 [34].

The age of people unaware of their HIV infection, often outside the traditional 'risk categories' (young men, either IVDUs or MSM), suggest that the traditional prevention strategies based exclusively on the awareness of belonging to a risk category or of having a risk behaviour are obsolete, despite recent WHO guidelines [35].

Positive samples were only found among males, despite the higher number of samples from females. This might be due to chance variation, considering the small numbers involved, or might reflect a lower prevalence among women of HIV infection in our province.

It is important to note that in our context, the positive predictive value of HIV Ab-testing was low, underscoring the need for correct pre- and post-test counselling to limit excessive anxiety at the screening test.

\section{Limitations}

Limitations of anonymous unlinked testing are well known: besides ethical issues [23], selection bias and lack of data on behaviour are the most important. In fact, in any community, the population accessing health care is not representative of the entire population; however, it is a convenient and low-cost sample [36]. Irreversible anonymisation prevents collection of important clinical and epidemiological information. However, especially in relatively small communities like Pavia province, from just a few data, it would be in theory possible to infer the personal identity of involved patients; we therefore choose to protect privacy over more detailed scientific information.

Because of anonymisation, patients with known HIV infection might have been included; however, to limit this bias, we excluded all samples taken from patients at the Infectious Disease Units.

Furthermore, some individuals might have been included twice in the sample: for negative samples, this would dilute the prevalence, and for positive, the opposite would be true; to counterbalance this, prior to anonymisation we excluded samples from the same patient within the previous 6 months.

We have not been able to quantify the HIV viral load in the positive samples; this is most likely due to the logistics of our study: we used plasma or serum samples discarded (and therefore used for our study) a week after the clinical use, a long time that does not allow the correct preservation of HIV-RNA.

Finally, we could not determine the CD4 cell count of positive samples, since testing was made on serum or plasma; it would have been interesting to assess the presumptive duration of HIV infection in these people.

\section{CONCLUSIONS}

Once obtained data on HIV prevalence in the general population, specific actions can be taken to inform, educate and counsel individuals on risk behaviours, HIV testing, antiretroviral therapy and preventative measures. We are now in the position to inform a wider public health programme.

While free and informed consent of each individual being tested is crucial, we believe that it is time to proceed from a strategy of HIV testing 'on demand' (such it is now) to one of active HIV-testing proposal by health-care workers.

Epidemiological data and sociological considerations demonstrate the evident need for developing new strategies for a wider and more accepted HIV testing in the general population. 


\section{CONFLICT OF INTEREST}

None.

\section{ETHICAL STANDARDS}

The authors assert that all procedures contributing to this work comply with the ethical standards of the relevant national and institutional committees on human experimentation and with the Helsinki Declaration of 1975 , as revised in 2008 .

However, the study design is observational only.

\section{DISCLOSURES}

The study was funded by the IRCCS Policlinic San Matteo Foundation. None of the authors has conflict of interest to declare.

\section{ACKNOWLEDGEMENTS}

The authors are greatly indebted to Giancarlo Iannello and Cinzia Ancarani of the Local Health Authority of Pavia, for data on known HIV prevalence and incidence of new diagnoses, and to Dr Sam Goblirsch, MD, for the careful English revision of the text. The study was supported by a research grant at IRCCS Policlinic San Matteo Foundation.

\section{REFERENCES}

1. Hall HI, Holtgrave DR, Maulsby C. HIV transmission rates from persons living with HIV who are aware and unaware of their infection. AIDS 2012; 26(7): 893-896.

2. Marks G, Crepaz N, Janssen RS. Estimating sexual transmission of HIV from persons aware and unaware that they are infected with the virus in the USA. AIDS 2006; 20(10): 1447-1450.

3. Brenner BG, et al. High rates of forward transmission events after acute/early HIV-1 infection. Journal of Infectious Diseases [Internet] 2007; 195: 951-959. Available from: http://www.ncbi.nlm.nih.gov/entrez/ query.fcgi?cmd $=$ Retrieve $\& \mathrm{db}=$ PubMed\&dopt $=$ Citation \&list_uids $=17330784$.

4. Ministro della Salute. Istituzione del sistema di sorveglianza delle nuove diagnosi di infezione da HIV. Decreto Ministeriale 31 marzo 2008. Gazzetta Ufficiale - Serie Generale [Internet] 2008; 175. Available from: http:// www.normativasanitaria.it/jsp/dettaglio.jsp?aggiornamenti $=\&$ attoCompleto $=$ si $\&$ id $=26058 \&$ page $=$ newsett $\&$ anno $=$ null.

5. Camoni L, et al. Aggiornamento delle nuove diagnosi di infezione da HIV e dei casi di AIDS in Italia al 31 dicembre 2013. Notiziario dell'Istituto Superiore di Sanità [Internet] 2014, 27(9 Supplemento 1), 3-47.
Available from: http://www.iss.it/binary/ccoa/cont/ ONLINE_6_COA_2014.pdf.

6. WHO, UNAIDS. Guidelines for Second Generation HIV Surveillance. Available from: http://www.who.int/ hiv/pub/surveillance/en/cds_edc_2000_5.pdf.

7. Branson BM, et al. Revised recommendations for HIV testing of adults, adolescents, and pregnant women in health-care settings. MMWR: Morbidity and Mortality Weekly Report 2006; 55(RR-14), 1-17; quiz CE1.

8. demo.istat.it/bilmens2016gen/query.php [Internet]. [cited 2016 Aug 26] Available from: http://demo.istat.it/bilmens2016gen/query.php?lingua $=i t a \& R i p=S 1 \& R e g=R 03$ \&submit=Tavola.

9. Raimondo M, et al. Caratteristiche delle persone che vivono con l'HIV e con l'AIDS in Italia. Notiziario dell' Istituto Superiore di Sanità [Internet] 2013; 26(7-8), 3-6. Available from: http://www.iss.it/binary/ccoa/cont/ Notiziario_ISS_vol_26_n_7_8_lug_ago_2013.pdf.

10. Regione Lombardia. SORVEGLIANZA DELLE MALATTIE INFETTIVE; anno 2015. 2016. Available from: http://www.welfare.regione.lombardia.it/shared/ ccurl/242/691/201620REPORT20SORVEGLIANZA20 DELLE20MALATTIE\%20INFETTIVE_2015.pdf.

11. Regione Lombardia. SORVEGLIANZA DELLE MALATTIE INFETTIVEIN LOMBARDIA:anno 2014. 2015. Available from: http://www.welfare.regione. lombardia.it/shared/ccurl/128/644/REPORT_MALATTI E\%20INFETTIVE_2014.pdf.

12. Revised guidelines on ethical and legal considerations in anonymous unlinked HIV seroprevalence research. Federal centre for AIDS working group on anonymous unlinked HIV seroprevalence research. Canadian Medical Association Journal 1992; 146(10): 1743-1746.

13. Wan L. The legality of unlinked anonymous screening for HIV infection: the U.S. approach. Health Policy 1990; 14(1): 29-35.

14. The unlinked anonymous HIV prevalence monitoring programme in England and Wales: preliminary results. CDR (London, England: Review) 1991; 1(7): R69-R76.

15. Bayer R, Lumey LH, Wan L. The American, British and Dutch responses to unlinked anonymous HIV seroprevalence studies: an international comparison. Law, Medicine \& Health Care 1991; 19(3-4): 222-230.

16. Larkin $\mathbf{C}$, et al. The unlinked anonymous HIV prevalence monitoring programme in N. Ireland 1992-1995. The Ulster Medical Journal 1997; 66(2); 96-99.

17. Postema EJ et al. Comparison of patients refusing with patients accepting unlinked anonymous HIV testing in an outpatient STD department in the Netherlands. International Journal of STD \& AIDS 1997; 8(6): 368-372.

18. Siedler A, Hamouda O, Schwartländer B. Anonymous unlinked testing as a sentinel approach: experience in Germany. Journal of Epidemiology and Community Health 1998; 52(Suppl. 1): 25S-27S.

19. Amar HS, Ho JJ, Mohan AJ. Human immunodeficiency virus prevalence in women at delivery using unlinked anonymous testing of newborns in the Malaysian setting. Journal of Paediatrics and Child Health 1999; 35(1): 63-66.

20. Rennie S, et al. Conducting unlinked anonymous HIV surveillance in developing countries: ethical, 
epidemiological, and public health concerns. PLoS Medicine 2009; 6(1): e4.

21. Rehle T, et al. Second-generation HIV surveillance: better data for decision-making. Bulletin of the World Health Organization 2004; 82(2): 121-127.

22. WHO. Ethical issues to be considered in second generation surveillance [Internet]. [cited 2004 Apr 27]. Available from: http://www.who.int/hiv/pub/epidemiology/en/sgs_ethical.pdf?ua $=1$.

23. Maher D. The ethics of feedback of HIV test results in population-based surveys of HIV infection. Bulletin of the World Health Organization 2013; 91(12): 950-956.

24. Garante Privacy. Autorizzazione generale al trattamento dei dati personali effettuato per scopi di ricerca scientifica. Gazzetta Ufficiale [Internet] 2012; 72. Available from: http://www.garanteprivacy.it/web/ guest/home/docweb/-/docweb-display/docweb/1878276.

25. Popolazione residente al $1^{\circ}$ gennaio [Internet]. [cited 2017 Jan 24]. Available from: http://dati.istat.it/Index. aspx?DataSetCode=DCIS_POPRES1.

26. Camoni L, et al. Estimates of the number of people living with HIV in Italy. BioMed Research International 2014; 2014: 209619.

27. Spectrum/EPP | UNAIDS [Internet]. [cited 2016 Sep 6] Available from: http://www.unaids.org/en/dataanalysis/ datatools/spectrumepp.

28. Mammone A et al. How many people are living with undiagnosed HIV infection? An estimate for Italy, based on surveillance data. AIDS 2016; 30(7): 1131-1136.

29. Hamers FF, Phillips AN. Diagnosed and undiagnosed HIV-infected populations in Europe. HIV Medicine 2008; 9(Suppl. 2): 6-12.
30. Op de Coul ELM, et al. Changing patterns of undiagnosed HIV infection in the Netherlands: who benefits most from intensified HIV test and treat policies? PLoS ONE 2015; 10(7): e0133232.

31. Moreno S et al. Prevalence of undiagnosed HIV infection in the general population having blood tests within primary care in Madrid, Spain. Sexually Transmitted Infections 2012; 88(7): 522-524.

32. Hall HI, et al. Prevalence of diagnosed and undiagnosed HIV infection - United States, 2008-2012. MMWR. Morbidity and Mortality Weekly Report 2015; 64(24): 657-662.

33. WHO | Towards universal access: Scaling up priority HIV/AIDS interventions in the health sector [Internet] [cited 2016 Mar 1]. Available from: http://www.who. int/hiv/pub/2009progressreport/en/.

34. WHO | WHO to develop guidance on HIV self-testing in 2016 [Internet] [cited 2016 Mar 2]. Available from: http://www.who.int/hiv/mediacentre/news/hiv-self-testing2016/en/.

35. Consolidated Guidelines on HIV Testing Services. 5Cs: Consent, Confidentiality, Counselling, Correct Results and Connection 2015. In: WHO Guidelines Approved by the Guidelines Review Committee. Geneva: World Health Organization, 2015.

36. Health Organization. Regional Office for Europe. W. Methods for second-generation HIV surveillance implementation for countries of Central and Eastern Europe (CEE) and the Baltic States : report on a WHO Workshop, Berlin Germany 27-29 May 2002 [Internet]. Copenhagen: WHO Regional Office for Europe, 2002. Available from: http:// www.euro.who.int/document/e77980.pdf. 